\title{
Computing Curie temperature of two-dimensional ferromagnets in the presence of exchange anisotropy
}

\author{
Sabyasachi Tiwari $\odot,{ }^{1,2,3}$ Joren Vanherck $\odot,{ }^{5,3}$ Maarten L. Van de Put, ${ }^{1}$ William G. Vandenberghe, ${ }^{1}$ and Bart Sorée $\odot^{3,4,5, *}$ \\ ${ }^{1}$ Department of Materials Science and Engineering, The University of Texas at Dallas, 800 West Campbell Road, \\ Richardson, Texas 75080, USA \\ ${ }^{2}$ Department of Materials Engineering, KU Leuven, Kasteelpark Arenberg 44, 3001 Leuven, Belgium \\ ${ }^{3}$ Imec, Kapeldreef 75, 3001 Heverlee, Belgium \\ ${ }^{4}$ Department of Electrical Engineering, KU Leuven, Kasteelpark Arenberg 10, 3001 Leuven, Belgium \\ ${ }^{5}$ Department of Physics, Universiteit Antwerpen, Groenenborgerlaan 171, 2020 Antwerp, Belgium
}

(Received 8 April 2021; accepted 7 September 2021; published 11 October 2021)

\begin{abstract}
We compare three first-principles methods of calculating the Curie temperature in two-dimensional (2D) ferromagnetic materials (FM), modeled using the Heisenberg model, and propose a simple formula for estimating the Curie temperature with high accuracy that works for all common 2D lattice types. First, we study the effect of exchange anisotropy on the Curie temperature calculated using the Monte Carlo (MC), the Green's function, and the renormalized spin-wave (RNSW) methods. We find that the Green's function method overestimates the Curie temperature in high-anisotropy regimes compared to the MC method, whereas the RNSW method underestimates the Curie temperature compared to the MC and the Green's function methods. Next, we propose a closed-form formula for calculating the Curie temperature of 2D FMs, which provides an estimate of the Curie temperature that is greatly improved over the mean-field expression for magnetic material screening. We apply the closed-form formula to predict the Curie temperature 2D magnets screened from the C2DB database and discover several high Curie temperature $\mathrm{FMs}$, with $\mathrm{Fe}_{2} \mathrm{~F}_{2}$ and $\mathrm{MoI}_{2}$ emerging as the most promising 2D ferromagnets. Finally, by comparing to experimental results for $\mathrm{CrI}_{3}, \mathrm{CrCl}_{3}$, and $\mathrm{CrBr}_{3}$, we conclude that for small effective anisotropies, the Green's-function-based equations are preferable, while for larger anisotropies, MC-based results are more predictive.
\end{abstract}

DOI: 10.1103/PhysRevResearch.3.043024

\section{INTRODUCTION}

Thanks to the recent discovery of the two-dimensional (2D) magnets $\mathrm{CrI}_{3}$ [1], $\mathrm{CrBr}_{3}$ [2], and $\mathrm{CrGeTe}_{3}$ [3], research in the field of $2 \mathrm{D}$ magnets has garnered unprecedented attention in the past few years. Their perceived application in spintronics [4,5], valleytronics [6], and skyrmion-based [7] magnetic memories [8] has sparked great interest. Moreover, the experimental demonstration of the electric field control of the magnetic order in $\mathrm{CrI}_{3}$ [1] provides a path towards the technological realization of electrically tunable magnetic memories using 2D magnets.

However, the low Curie temperature of 2D magnets acts as a hurdle in their practical application. Most of the 2D magnets discovered experimentally have a low Curie temperature, e.g., $45 \mathrm{~K}$ for $\mathrm{CrI}_{3}$ [1] and $34 \mathrm{~K}$ for $\mathrm{CrBr}_{3}$ [2]. While $\mathrm{Fe}_{3} \mathrm{GeTe}_{2}$ has a Curie temperature of $130 \mathrm{~K}$ [9], it has an itinerant magnetic behavior, which cannot be controlled using an external electric

\footnotetext{
*bart.soree@imec.be

Published by the American Physical Society under the terms of the Creative Commons Attribution 4.0 International license. Further distribution of this work must maintain attribution to the author(s) and the published article's title, journal citation, and DOI.
}

field. On the other hand, the high Curie temperature in $\mathrm{VSe}_{2}$ [10] is a matter of debate, with reports emerging of $\mathrm{VSe}_{2}$ having a charge-density-wave ground state with no magnetic ordering $[11,12]$.

The dearth of high Curie temperature 2D magnets has led to an unprecedented effort in the search for 2D magnets with higher Curie temperature. Thankfully, the possible span of 2D magnets is quite large, starting from 2D crystals [13] to conventional $2 \mathrm{D}$ materials doped with transition metals [14-19]. However, experiments can only be performed for the most promising 2D ferromagnets. Hence, a vast amount of research is dedicated to high-throughput screening of the most promising 2D magnets from theory [20-22].

A common strategy in such high-throughput calculations for predicting the Curie temperature of $2 \mathrm{D}$ magnets is as follows. First, obtain 2D materials with magnetic ordering from material databases such as the C2DB [23]. Then, approximate the magnetic structure using a parameterized Heisenberg model whose parameters are obtained from the density functional theory (DFT) calculations [24]. Finally, predict the Curie temperature from the phase change of the Heisenberg Hamiltonian, calculated using computationally costly Monte Carlo simulations with anisotropy [20-22,25] or even using the Ising model [13]. The Monte Carlo simulations are costly in terms of computational time and memory compared to the mean-field theory. As a result, many researchers use the 
less accurate mean-field calculations for predicting the Curie temperature of newly discovered materials $[25,26]$.

Monte Carlo simulations with anisotropy result in a rather accurate estimation of the Curie temperature for most of the experimentally verified 2D magnets yet discovered [24,27]. However, care must be taken because the calculated Curie temperature depends on the parameters of the Heisenberg Hamiltonian [28] and on the approximation used at the DFT level. Moreover, the recent application of methods that take into account the quantum mechanical fluctuations in the Heisenberg model to 2D magnets, e.g., the Green's function $[29,30]$ and the renormalized spin-wave [31] methods, raises further questions on how much the Curie temperature depends on the level of approximation used to solve the Heisenberg Hamiltonian. Moreover, the Curie temperature of 2D ferromagnets strongly depends on the anisotropy [32], which is itself dependent on the spin-orbit interaction of the material [31,33-35]. The different methods used to solve the Heisenberg Hamiltonian have a different impact of anisotropy. So, it is highly desirable to understand how much the Curie temperature of the 2D ferromagnets depends on various methods used for solving the Heisenberg Hamiltonian. There have been previous works on understanding the impact of exchange anisotropy on the Curie temperature of 2D magnets. Most of the works have either focused on using only the MC simulations to obtain a closed-form description [25] or have compared the Curie temperature using different methods in extremely high regimes of the exchange anisotropy [27].

We compare, for 34 2D materials, three methods of calculating the Curie temperature from a Heisenberg Hamiltonian: the Monte Carlo (MC), Green's function, and renormalized spin-wave (RNSW) methods. We first provide a brief overview of the Heisenberg Hamiltonian, while the three solution methods are discussed elaborately in the Appendix. Next, we provide an analytical formula to approximate the Curie temperature calculated using the three solution methods, as a function of nearest-neighbor exchange strength $(J)$ and anisotropy $\left(\Delta_{\mathrm{NN}}\right)$. Further, we calculate the Curie temperature using the three methods, as a function of exchange anisotropy, and fit the analytical formula to each. We then calculate the Curie temperature of 34 2D ferromagnets screened from the C2DB database [23] using our analytical formula and find some very promising ferromagnets with high Curie temperature, including $\mathrm{Fe}_{2} \mathrm{~F}_{2}$ and $\mathrm{MoI}_{2}$ having, for all methods, estimations above 403 and $281 \mathrm{~K}$, respectively. Finally, we show that the Curie temperature calculated using the three methods depends quantitatively on the long-range interactions; however, the qualitative trend remains the same and the analytical formula we develop provides a good estimation for a first-level theoretical screening.

\section{METHODOLOGY}

\section{A. The Heisenberg Hamiltonian}

Two-dimensional ferromagnets are most commonly modeled through the Heisenberg Hamiltonian,

$$
H=\frac{1}{2} \sum_{i, j} \hat{\mathbf{S}}_{i} \boldsymbol{J}_{i j} \hat{\mathbf{S}}_{j}+\sum_{i} D\left(\hat{S}_{i}^{z}\right)^{2},
$$

where $\hat{\mathbf{S}}=\hat{S}^{x} \mathbf{x}+\hat{S}^{y} \mathbf{y}+\hat{S}^{z} \mathbf{z}$ is the spin operator. Here, the spin operator can take eigenvalues $S=n / 2$, with $n$ a strictly positive integer. The off-diagonal elements of the $J$ tensor $\boldsymbol{J}_{i j}$ between spins at sites $i$ and $j$ have been found to be much smaller than the diagonal elements for most of the 2D ferromagnets (FMs), and hence, we assume the off-diagonal elements to be zero when modeling ferromagnets. For the present study, exchange interactions up to second neighbor are accounted for, while the second term of the Heisenberg Hamiltonian - called the on-site anisotropy-is ignored. The Heisenberg Hamiltonian thus reduces to

$$
\begin{aligned}
H & =\frac{1}{2} \sum_{i, j} \hat{S}_{i}^{x} J_{i j}^{x x} \hat{S}_{j}^{x}+\hat{S}_{i}^{y} J_{i j}^{y y} \hat{S}_{j}^{y}+\hat{S}_{i}^{z} J_{i j}^{z z} \hat{S}_{j}^{z} \\
& =\sum_{i, j} \frac{J_{i j}}{2}\left[\hat{\mathbf{S}}_{i} \cdot \hat{\mathbf{S}}_{j}+\Delta_{i j}\left(\hat{S}_{i}^{z} \hat{S}_{j}^{z}-\hat{S}_{i}^{x} \hat{S}_{j}^{x}-\hat{S}_{i}^{y} \hat{S}_{j}^{y}\right)\right],
\end{aligned}
$$

where the anisotropy is modeled through distinct values for the in-plane and out-of-plane anisotropic exchange strength, respectively; $J^{x x}=J^{y y}=J(1-\Delta)$ and $J^{z z}=J(1+\Delta)$. The unitless anisotropy $\Delta_{i j}=\left(J_{i j}^{z z}-J_{i j}^{x x}\right) / 2 J_{i j}$ is said to be of the easy-axis type when positive, while of the easy-plane type otherwise.

We solve the Heisenberg Hamiltonian as a function of temperature using the MC, the Green's function, and the RNSW methods. These three methods used to solve the Heisenberg Hamiltonian are discussed extensively in the Appendix.

\section{B. Analytical formula for screening 2D magnets}

Using the exact methods of calculating the Curie temperature, i.e., the MC, the Green's function, and the RNSW methods, is computationally costly. Hence, to provide a closed-form equation for $T_{\mathrm{C}}$ calculated using all three methods, we propose the analytical formula

$$
T_{\mathrm{C}}=\frac{1}{\alpha_{1}-\alpha_{2} \ln \left(\Delta_{\mathrm{NN}}\right)} \frac{J\left(S^{2}+\theta S\right)}{k_{\mathrm{B}}},
$$

which is inspired by the group-theoretical approach used by Bander et al. [36]. Here, $S$ is the spin eigenvalue and $\Delta_{\mathrm{NN}}$ is the nearest-neighbor exchange anisotropy. $k_{\mathrm{B}}$ is the Boltzmann constant. $\theta=1$ for the Green's function and RNSW methods, and $\theta=0$ for the MC method. Dimensionless parameters $\alpha_{1}$ and $\alpha_{2}$ are fit so that $T_{\mathrm{C}}$ matches the Curie temperature as a function of $\Delta_{\mathrm{NN}}, J$, and $S$, obtained using the MC, Green's function, and RNSW methods for the hexagonal, honeycomb, and square lattices (Fig. 1).

The anisotropy originates due to the spin-orbit coupling, and spin-orbit coupling is a much weaker interaction than the electronic exchange interaction. $\Delta_{\mathrm{NN}}$ is the ratio of the strength of the anisotropy and exchange interaction $J$; it is highly unlikely that $\Delta_{\mathrm{NN}}$ would approach 1 for any 2D magnetic material. Nevertheless, we fit the formula only up to $\Delta_{\mathrm{NN}}=0.2$ to the exact methods. Also, the group-theoretical method applied by Bander et al. [36] is only valid around $\Delta_{\mathrm{NN}} \rightarrow 0$, from which our formula is inspired. Therefore, the formula should only be used to calculate the Curie temperature of materials whose $\Delta_{\mathrm{NN}} \leqslant 0.2$. 

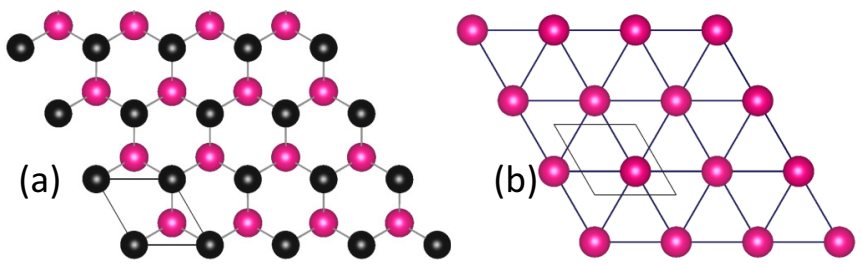

(c)

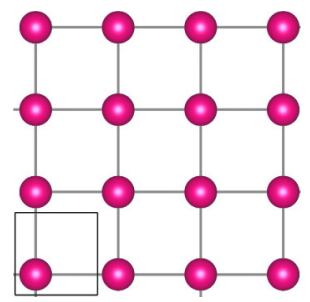

(d)

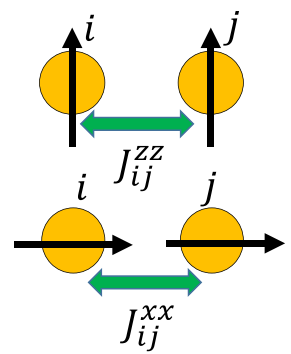

FIG. 1. Three different type of lattices for 2D materials: (a) honeycomb, (b) hexagonal, and (c) square lattices. For the (b) hexagonal and the (c) square lattices, the unit cell contains only one atom, whereas for the (a) honeycomb lattice, the unit cell has two atoms, referred to as sublattice $A$ (pink) and sublattice $B$ (black). (d) The in-plane $\left(J_{i j}^{x x}\right)$ and out-of-plane $\left(J_{i j}^{z z}\right)$ exchange interaction between spins $i$ and $j$.

\section{Obtaining the input parameters of the analytical formula}

The parameters for the analytical formula proposed in Eq. (3) can be obtained from DFT total energy calculations using

$$
\begin{aligned}
J^{\perp} & =\frac{E_{\mathrm{FM}}^{\perp}-E_{\mathrm{AFM}}^{\perp}}{2 N_{\mathrm{NN}} S^{2}}, \\
J^{\|} & =\frac{E_{\mathrm{FM}}^{\|}-E_{\mathrm{AFM}}^{\|}}{2 N_{\mathrm{NN}} S^{2}}, \\
J & =\frac{J^{\perp}+J^{\|}}{2}, \\
\Delta_{\mathrm{NN}} & =\frac{J^{\perp}-J^{\|}}{2 J} .
\end{aligned}
$$

Here, $E_{\mathrm{FM}}^{\perp / \|}$ and $E_{\mathrm{AFM}}^{\perp / \|}$ are the total energies calculated using the DFT for FM and antiferromagnet (AFM) order with the magnetic axis oriented in the out-of-plane/in-plane direction. $N_{\mathrm{NN}}$ is the number of nearest neighbors. For obtaining parameters beyond nearest neighbor, one has to use the advanced mapping methods presented in Ref. [24].

\section{RESULTS AND DISCUSSION}

We first discuss the impact of nearest-neighbor exchange anisotropy on the Curie temperature calculated using the three exact methods, and fit to this the $T_{\mathrm{C}}$ calculated using the analytical formula. We then calculate the Curie temperature of 2D ferromagnets screened from the C2DB database using the fitted analytical formula. Finally, we discuss the impact of next-nearest-neighbor anisotropy on the Curie temperature of $\mathrm{Cr}$ compounds.

\section{A. Effect of exchange anisotropy}

Figure 2(a) shows the calculated Curie temperature $\left(T_{\mathrm{C}}\right)$ for a hexagonal 2D material as a function of nearest-neighbor exchange anisotropy $\left(\Delta_{\mathrm{NN}}\right)$ using the three methods. For all three methods, the Curie temperature increases with increasing anisotropy. Moreover, for zero anisotropy, the Curie temperature tends to zero for all three methods.

There are three regions with a distinct feature in Fig. 2(a). First, within the yellow shaded region, the Curie temperature calculated using the MC and the Green's function methods matches closely. For lower anisotropies, the Green's function approach results in a Curie temperature that is lower compared to the MC approach, whereas for higher anisotropies, the situation is opposite: the Curie temperature estimated using the Green's function is higher than the one estimated using the MC method. Moreover, for all three regions, the renormalized spin-wave approach results in a Curie temperature below both the MC and the Green's function methods.

The existence of three regions for the MC and the Green's function methods can be understood by careful observation of the exact Curie temperature formulas. In the limit of $\Delta_{\mathrm{NN}} \rightarrow$ 1 , the Curie temperature calculated using the Green's function method tends to the result from the molecular-field theory [29], which overestimates the Curie temperature, whereas for higher anisotropies $\Delta_{\mathrm{NN}} \rightarrow 1$, the Curie temperature calculated using the MC method remains below the Ising limit [27]. Therefore, because the molecular-field theory results in an overestimation of the Curie temperature compared to the Ising model, the Green's function method overestimates the Curie temperature compared to the $\mathrm{MC}$ method at higher anisotropies. For $\Delta_{\mathrm{NN}}=0$, the Curie temperature calculated using the Green's function method goes to zero, in accordance with the Mermin-Wagner theorem [32], whereas for the MC method, the average magnetization $M=\sqrt{S_{x}^{2}+S_{y}^{2}+S_{z}^{2}}$ remains finite due to the nonzero exchange interaction. Hence, for lower anisotropies, the MC method overestimates the Curie temperature.

To further understand the impact of $\Delta_{\mathrm{NN}}$, we plot the sensitivity $\left(\frac{1}{T_{\mathrm{C}}} \frac{d T_{\mathrm{C}}}{d \Delta_{\mathrm{NN}}}\right)$ of the Curie temperature in Fig. 2(b) for all three methods. We observe that both the RNSW and Green's function methods have an almost similar sensitivity to $\Delta_{\mathrm{NN}}$, and the sensitivity decreases with increasing anisotropy. Moreover, we see that the MC method is much less sensitive to $\Delta_{\mathrm{NN}}$, especially at lower anisotropy compared to the RNSW and Green's function methods.

\section{B. Fitting of analytical formula to exact methods}

Figure 2 also shows the fit of the analytical function [Eq. (3)] to the Curie temperature calculated using the MC, the Green's function, and the RNSW method, respectively, for a honeycomb lattice. The corresponding figures for the hexagonal lattice and the square lattice are provided in the Supplemental Material [37]. For all three lattices, we use $\Delta_{\mathrm{NN}}=0.0$ to $\Delta_{\mathrm{NN}}=0.2$ as the fitting range. We observe that Eq. (3) fits remarkably well to the Curie temperature calculated using the mentioned methods. The parameters $\alpha_{1}$ and $\alpha_{2}$ are provided in Table I. From Table I, we see that the parameter $\alpha_{2}$ for the MC method is much lower compared to the Green's function and RNSW methods. 


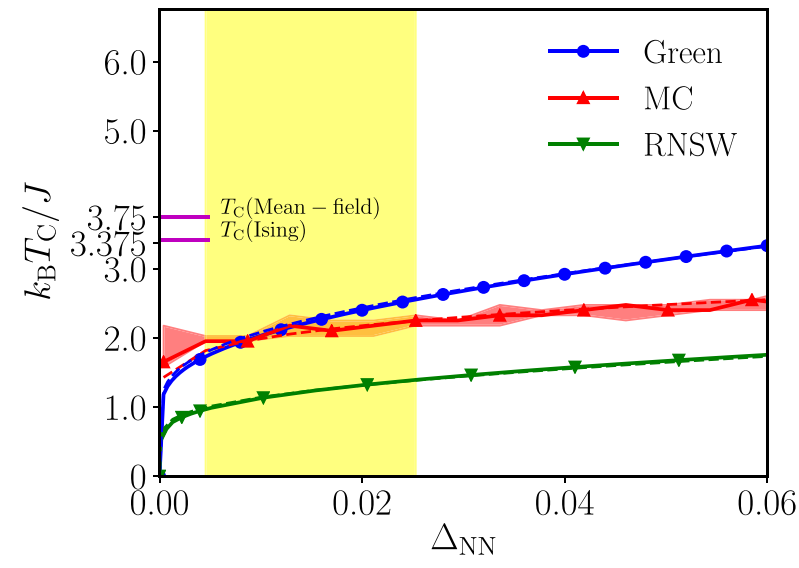

(a)

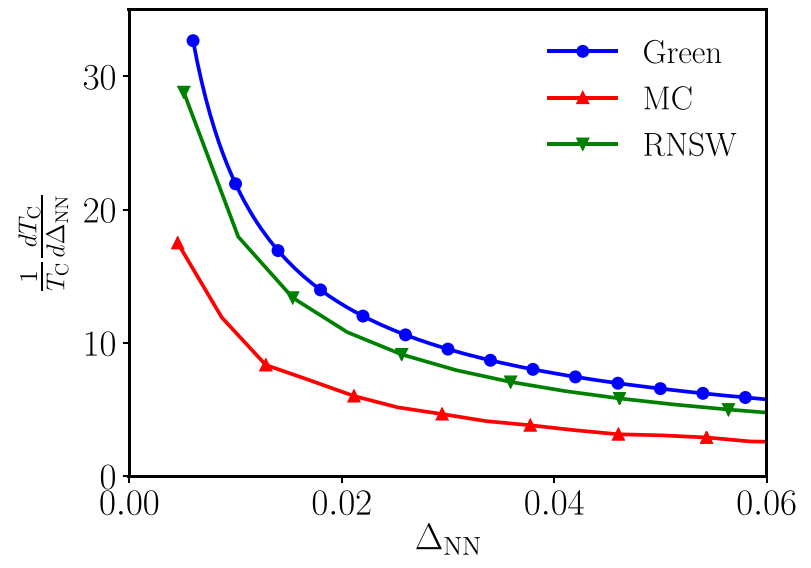

(b)

FIG. 2. (a) Comparison of Curie temperature calculated using the Green's function, the Monte Carlo (MC), and the renormalized spin-wave (RNSW) methods as a function of nearest-neighbor exchange anisotropy $\left(\Delta_{\mathrm{NN}}\right)$ for a honeycomb lattice. For the MC method, the solid line shows the median and the shading shows the 25 th to 75 th percentile of the calculated Curie temperature. The dashed lines show a fit using function $\left[\alpha_{1}-\alpha_{2} \ln \left(\Delta_{\mathrm{NN}}\right)\right]^{-1} J\left(S^{2}+\theta S\right) / k_{\mathrm{B}}$ for the respective methods. The horizontal tick shows the Ising limit $\left(1.52 S^{2}\right.$, with $\left.S=3 / 2\right)$ and the quantum mean field $\left(1 / 3\left[S(S+1) N_{\mathrm{NN}}\right], N_{\mathrm{NN}}=3\right.$ for honeycomb lattice). The yellow shaded area shows the region where the MC and the Green's function methods have a difference of less than 10\%. (b) Comparison of Curie temperature sensitivity calculated using the Green's function, the MC, and the RNSW methods as a function of nearest-neighbor exchange anisotropy $\left(\Delta_{\mathrm{NN}}\right)$ for a honeycomb lattice.

\section{Screening of 2D magnets from C2DB and their critical temperatures}

Figure 3(a) shows a schematic of our screening process. We screen the C2DB database [23] for ferromagnetic 2D materials with out-of-plane exchange anisotropy $(J>0$ and $\Delta_{\mathrm{NN}}>0$ ). We find $342 \mathrm{D}$ magnets with FM order in their ground state. Next, we calculate their Curie temperature using the analytical formula in Eq. (3).

Figures 3(b) and 3(c) show the calculated Curie temperature of the screened ferromagnets sorted as a function of exchange interaction $\left(J_{i j}\right)$ and exchange anisotropy $\left(\Delta_{\mathrm{NN}}\right)$, calculated using the MC, the Green's function, and the RNSW methods. The table comprising the Curie temperature using the different methods is provided in the Supplemental Material [37].

We observe some general trends from Figs. 3(b) and 3(c). First, the Curie temperature is indeed dependent on the methods used to solve the Heisenberg Hamiltonian. We observe the same pattern as in Fig. 2(a), where the Curie temperature calculated using the RNSW method remains low for all the materials; however, for higher anisotropy, the RNSW method starts approaching the MC results. Given that all of the identified 2D ferromagnets from the C2DB database have anisotropy, i.e., $\Delta_{\mathrm{NN}}<0.25$, we can say that the RNSW

TABLE I. Parameters of the analytical formula.

\begin{tabular}{lcccc}
\hline \hline Lattice & Parameter & MC & Green & RNSW \\
\hline \multirow{2}{*}{ Honeycomb } & $\alpha_{1}$ & 0.49 & 0.07 & 0.40 \\
& $\alpha_{2}$ & 0.14 & 0.37 & 0.62 \\
Hexagonal & $\alpha_{1}$ & 0.24 & 0.24 & 0.32 \\
& $\alpha_{2}$ & 0.045 & 0.14 & 0.21 \\
Square & $\alpha_{1}$ & 0.37 & 0.34 & 0.43 \\
& $\alpha_{2}$ & 0.08 & 0.24 & 0.36 \\
\hline \hline
\end{tabular}

method gives the most conservative estimation of the Curie temperature. On the other hand, the Curie temperature calculated using the Green's function method remains below the MC method for compounds until $\mathrm{ZrI}_{2}\left(\Delta_{\mathrm{NN}}=0.0072\right)$. Most remarkably, from our screening using the analytical formulas for all three methods, we identify that some very promising candidates for realizing 2D ferromagnets are $\mathrm{Fe}_{2} \mathrm{~F}_{2}$ and $\mathrm{MoI}_{2}$ for whom even the RNSW method predicts a Curie temperature of 403 and $281 \mathrm{~K}$, respectively. $\mathrm{Fe}_{2} \mathrm{~F}_{2}$ has a high exchange interaction strength that leads to its higher Curie temperature, whereas $\mathrm{MoI}_{2}$ has a higher exchange anisotropy that leads to a higher Curie temperature.

Interestingly, we see from Figs. 3(b) and 3(c) that all three experimentally discovered $\mathrm{Cr}$ compounds, i.e., $\mathrm{CrI}_{3}, \mathrm{CrBr}_{3}$, and $\mathrm{CrCl}_{3}$, are screened from the C2DB database. For $\mathrm{CrI}_{3}$, we obtain a Curie temperature of 41,31 , and $23 \mathrm{~K}(45 \mathrm{~K}$, experimental [1]), and for $\mathrm{CrBr}_{3}$, we find a Curie temperature of 26,24 , and $14 \mathrm{~K}$ (34 K, experimental [2]) from the Green's function, the MC, and the RNSW method, respectively. The close estimation of the Curie temperature of $\mathrm{Cr}$ compounds compared to their experimental values suggests that the analytical formula in Eq. (3) can be used for an efficient first-level screening of 2D ferromagnetic compounds.

It should be noted that the obtained high Curie temperature for metals, e.g., $\mathrm{Fe}_{2} \mathrm{~F}_{2}$, using our formulas provides a rough first-level estimate because their itinerant magnetic nature is not fully captured by the Heisenberg model. Therefore, a more detailed approach is needed to precisely predict their magnetic order as a function of temperature, e.g., dynamical mean-field theory.

\section{Impact of next-nearest-neighbor anisotropy}

Until now, we discussed the impact of nearest-neighbor exchange anisotropy on the Curie temperature calculated using various methods and showed our analytical formula, 


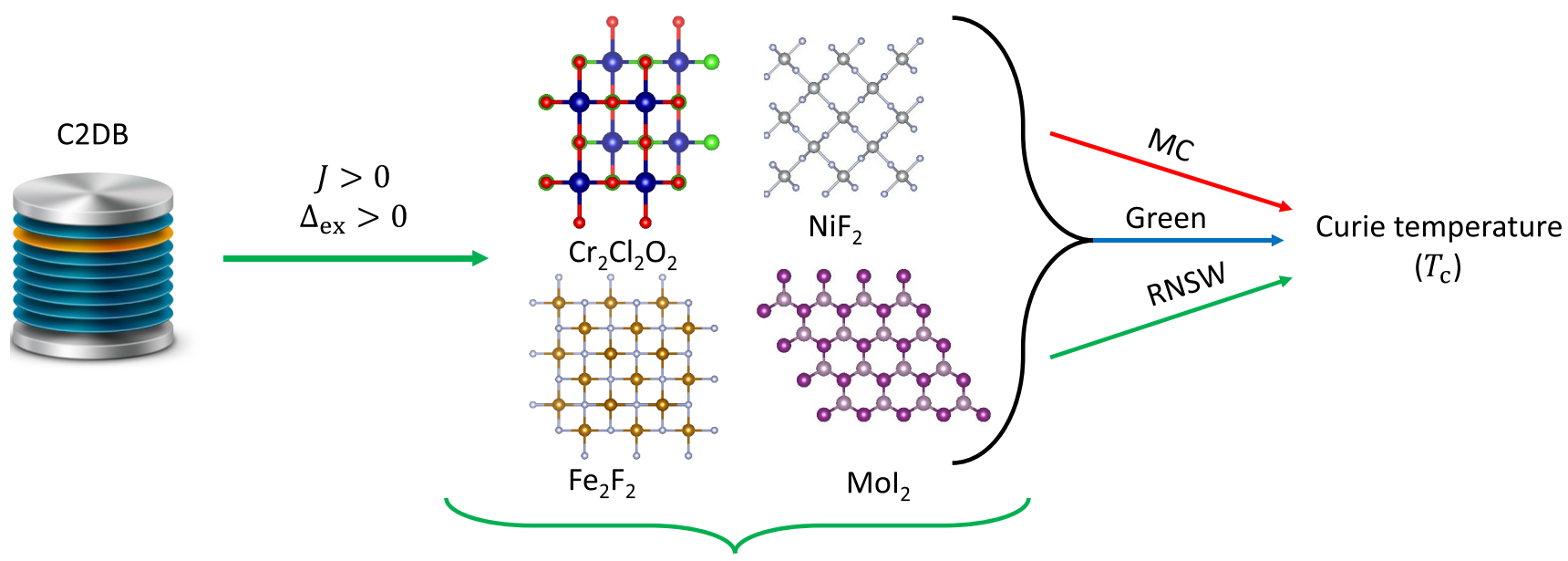

34 screened 2D materials

(a)

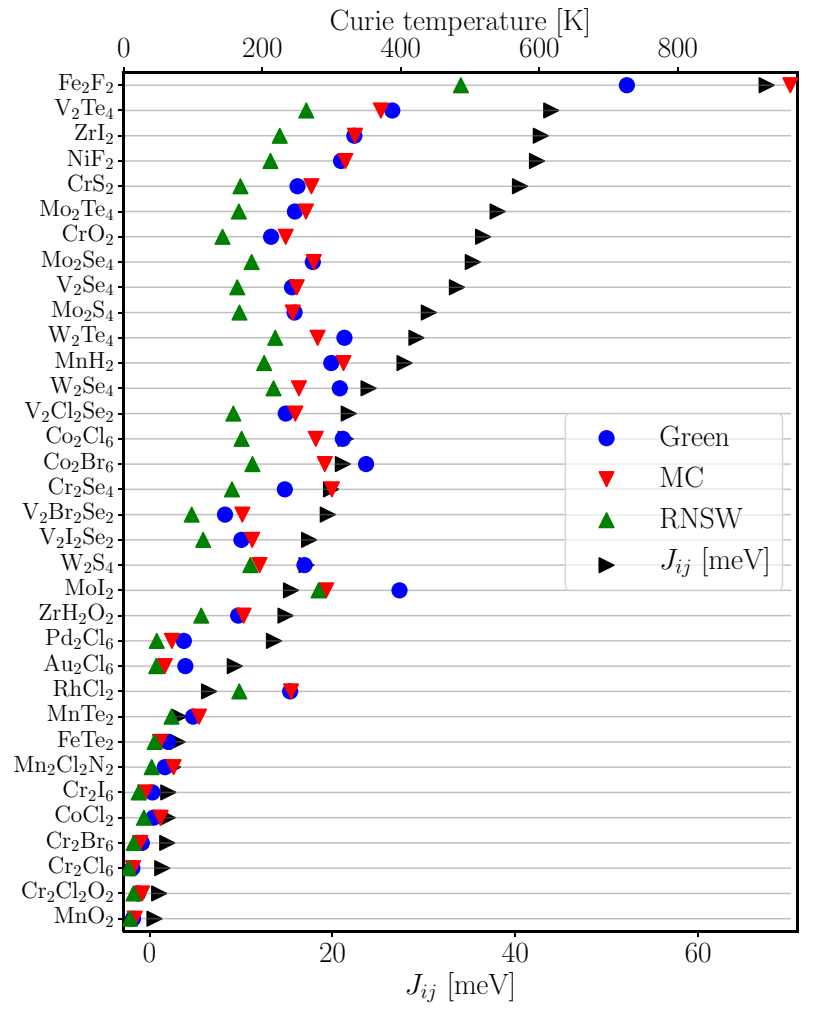

(b)

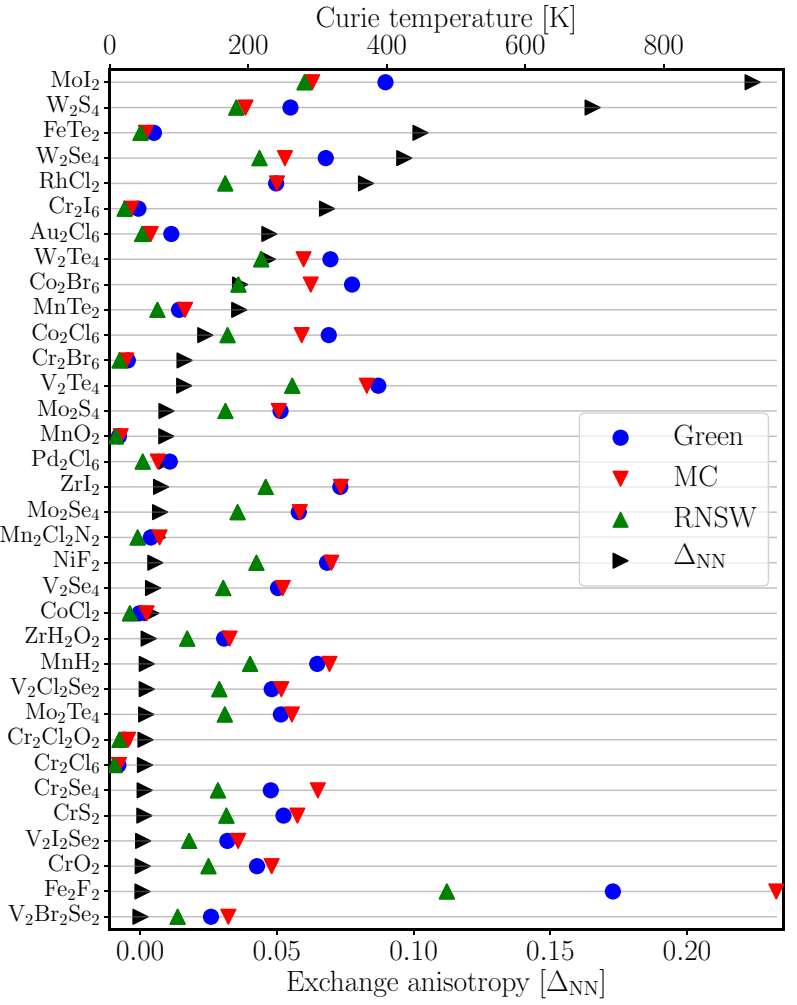

(c)

FIG. 3. (a) Materials obtained from the C2DB database with positive out-of-plane anisotropy and positive exchange interaction. The Curie temperature of the screened materials using the analytical formula for the MC, the Green's function, and the RNSW (top abscissa) methods sorted as a function of (b) exchange interaction $\left(J_{i j}\right)$ and (c) exchange anisotropy $\left(\Delta_{\mathrm{NN}}\right)$.

used for high-throughput screening of 2D FMs. Here, we discuss the impact of next-nearest-neighbor interactions, especially the next-nearest-neighbor anisotropy, and the impact of long-range interactions on the theoretical prediction of Curie temperature of 2D FMs. We use the honeycomb lattice as an example.

Figure 4 shows the comparison between the Curie temperature $\left(T_{\mathrm{C}}\right)$ for a honeycomb $2 \mathrm{D}$ material as a function of next-nearest-neighbor exchange anisotropy $\left[\Delta_{\mathrm{NNN}}=\right.$
$\left.\left(J_{\mathrm{NNN}}^{z}-J_{\mathrm{NNN}}^{x}\right) /\left(2 J_{\mathrm{NNN}}\right)\right]$ for a honeycomb lattice. We keep the nearest-neighbor anisotropy fixed at $\Delta_{\mathrm{NN}}=0.01$ and nearest-neighbor exchange at $J_{\mathrm{NN}}=2.5 \mathrm{meV}$. We observe that both the Green's function and RNSW methods have a similar sensitivity to the next-nearest-neighbor anisotropy, whereas the MC calculations are relatively less sensitive. As discussed in Sec. III A, the observed low sensitivity of the MC simulations to next-nearest-neighbor anisotropy is due to their classical nature. 


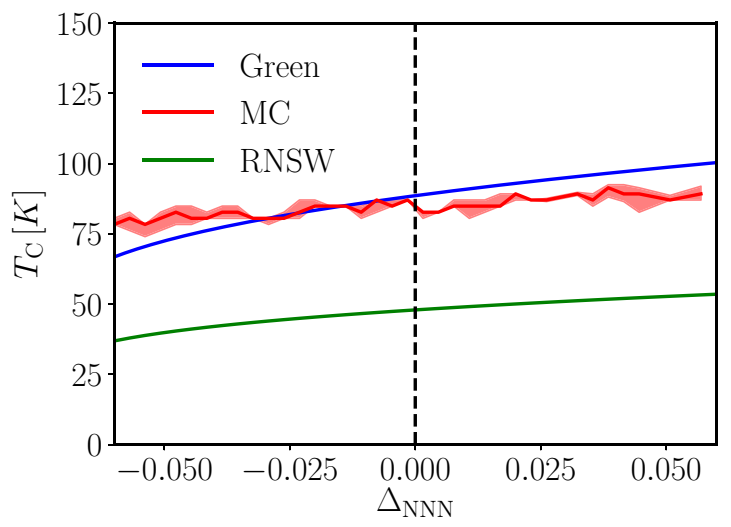

FIG. 4. Comparison of Curie temperature calculated using the Green's function, Monte Carlo, and the renormalized spin-wave methods as a function of next-nearest-neighbor exchange anisotropy $\left(\Delta_{\mathrm{NNN}}\right)$ for a honeycomb lattice. The vertical dashed line shows the $\Delta_{\mathrm{NNN}}=0$ at which Fig. 2 is calculated. For the MC method, the solid line shows the median and the shading shows the 25th to 75th percentile of the calculated Curie temperature.

\section{E. Importance of long-range interactions for the quantitative estimation of $T_{\mathrm{C}}$}

While the approximate results of the analytical formula are good as a first screening, further neighbors can still lead to significant changes of the $T_{\mathrm{C}}$. We compare the Curie temperature calculated for $\mathrm{Cr}$ compounds, i.e., $\mathrm{CrI}_{3}, \mathrm{CrBr}_{3}, \mathrm{CrCl}_{3}$, and $\mathrm{CrGeTe}_{3}$, using the $\mathrm{MC}$, the Ising $\mathrm{MC}$, the Green's function, and the RNSW methods, to their experimentally measured value in Table II, including the long-range interactions. The experimental comparison of methods is subject to the parameters obtained from DFT and with a change in exchange functional, i.e., the $J$ parameters change. However, the Perdew-Burke-Ernzerhof (PBE) parameters have been found to be close to experiments for $\mathrm{CrI}_{3}$ [38] and serve as a good benchmark for evaluating the three methods and their regimes of applicability.

We observe that the Curie temperature calculated using the MC and the RNSW methods is in good agreement with the experimental values for $\mathrm{CrI}_{3}$, and $\mathrm{MC}$ and the Green's function methods is in good agreement for $\mathrm{CrBr}_{3}$, whereas the Curie temperature calculated using the RNSW and the Green's function methods results in a good agreement for $\mathrm{CrCl}_{3}$. The Curie temperature of monolayer $\mathrm{CrGeTe}_{3}$ has not been reported experimentally yet; however, if we consider that the Curie temperature follows the same trend as reported in [3], we find that the RNSW method results in a much closer estimation of the Curie temperature. It is worth noting that the experimental Curie temperature for all three Cr-compounds

TABLE II. Curie temperature (K) of $\mathrm{Cr}$ compounds using exact methods with long-range interactions.

\begin{tabular}{|c|c|c|c|c|c|c|}
\hline Method & $\mathrm{MC}$ & Green & RNSW & Ising & Mean-field (NN) & Expt. \\
\hline $\mathrm{CrI}_{3}$ & 63 & 83 & 36 & 181 & 89 & $45[1]$ \\
\hline $\mathrm{CrBr}_{3}$ & 37 & 39 & 20 & 130 & 55 & $35[2]$ \\
\hline $\mathrm{CrCl}_{3}$ & 25 & 21 & 15 & 100 & 53 & 17 (bulk) [39] \\
\hline $\mathrm{CrGeTe}_{3}$ & 64 & 68 & 38 & 314 & 237 & 42 (bilayer) [3] \\
\hline
\end{tabular}

TABLE III. $J$ parameters and anisotropies of experimental $\mathrm{Cr}$ compounds.

\begin{tabular}{lcrlcll}
\hline \hline Parameters & $\begin{array}{c}J_{\mathrm{NN}} \\
(\mathrm{meV})\end{array}$ & $\begin{array}{c}J_{\mathrm{NNN}} \\
(\mathrm{meV})\end{array}$ & $\begin{array}{c}J_{\mathrm{NNNN}} \\
(\mathrm{meV})\end{array}$ & $\Delta_{\mathrm{NN}}$ & $\Delta_{\mathrm{NNN}}$ & $\Delta_{\mathrm{NNNN}}$ \\
\hline $\mathrm{CrI}_{3}$ & 2.21 & 0.75 & & 0.029 & 0.043 & \\
$\mathrm{CrBr}_{3}$ & 1.38 & 0.44 & & 0.010 & 0.012 & \\
$\mathrm{CrCl}_{3}$ & 1.31 & 0.24 & & 0.001 & 0.006 & \\
$\mathrm{CrGeTe}_{3}$ & 5.87 & -0.28 & 0.345 & 0.02 & 0.0 & 0.028 \\
\hline \hline
\end{tabular}

lies between what is calculated by the three methods with RNSW setting the lower limit and Green's function and MC setting the upper limit.

We now compare the $J$ parameters for the $\mathrm{Cr}$ compounds shown in Table III to the Curie temperatures calculated using the various methods in Table II. From Table III, we find the nearest-neighbor anisotropy $\Delta_{\mathrm{NN}}=0.029$ for $\mathrm{CrI}_{3}$. As expected from Fig. 2, the MC Curie temperature is below the Green's function Curie temperature for $\mathrm{CrI}_{3}$. For $\mathrm{CrBr}_{3}$, the nearest-neighbor anisotropy $\Delta_{\mathrm{NN}}=0.01$, which is in the range where the MC and Green's function results almost overlap in Fig. 2. Hence, the Curie temperature calculated using the MC method is almost the same as the one calculated using the Green's function method. For $\mathrm{CrCl}_{3}$, the nearest-neighbor anisotropy $\Delta_{\mathrm{NN}}=0.001$, which is in the range where the RNSW and Green's function methods are closer and the MC method overestimates the Curie temperature. Hence, the Curie temperature calculated using the MC method is higher than the Green's function method. For $\mathrm{CrGeTe}_{3}$, the anisotropy is in the range where the MC and the Green's function method have a difference less than 10\% [from Fig. 2(a)], and hence, the Curie temperature calculated using the MC and Green's function methods is almost the same.

We see in Table II that the inclusion of the next-nearestneighbor interaction does impact the Curie temperature quantitatively. A similar conclusion was drawn in our previous works for both bulk [24] and monolayer 2D magnets [30]. However, the trend of Fig. 3 remains the same, with the RNSW method being the most conservative, and the MC and Green's function methods interchanging their estimation depending on the anisotropy. Therefore, the analytical formulas provided in this work can be used to obtain a qualitative estimation of Curie temperatures of 2D ferromagnets with the least effort. However, for quantitatively more accurate results, one will have to include the long-range interactions.

\section{CONCLUSION}

We have compared three common methods, i.e., the MC, the Green's function, and the RNSW methods, used for calculating the Curie temperature of 2D ferromagnets, which are modeled using the Heisenberg Hamiltonian. We have investigated the impact of nearest-neighbor exchange anisotropy as well as the next-nearest-neighbor anisotropy on the Curie temperature calculated using the mentioned methods. We have shown that the Curie temperature calculated using the Green's function and the MC methods as a function of nearest-neighbor anisotropy results in three regions. At low anisotropy, the Green's function method results in a lower Curie temperature; at higher anisotropy, the MC method 
results in a lower Curie temperature; and in-between, there is a region where both the Green's function and the MC methods result in the same Curie temperature estimation.

We have provided a closed-form analytical formula to calculate the Curie temperature of 2D FMs using nearestneighbor exchange and anisotropy. The analytical formula to calculate the Curie temperature has been fitted to the exact Curie temperature obtained from the MC, the Green's function, and the RNSW methods. We have applied our formula on 34 2D ferromagnets screened from the C2DB database [23] and found some very promising ferromagnets with high Curie temperature, including $\mathrm{Fe}_{2} \mathrm{~F}_{2}$, and $\mathrm{MoI}_{2}$ which have the lowest estimation of 403 and $281 \mathrm{~K}$, respectively.

By comparing the Curie temperature of the experimentally grown $\mathrm{Cr}$ compounds, we found that for $\mathrm{CrCl}_{3}$, which has low anisotropy, the RNSW and the Green's function methods result in a more accurate estimation of the Curie temperature, whereas for $\mathrm{CrI}_{3}$, which has a higher anisotropy, the MC method results in a good estimation of the Curie temperature. Moreover, for $\mathrm{CrBr}_{3}$, which has an intermediate anisotropy, both the MC and the Green's function methods result in a similar estimation of the Curie temperature, suggesting that for materials with low anisotropy, the RNSW and the Green's function methods will result in a more accurate description of the Curie temperature, whereas for materials with higher anisotropy, the MC method results in a better estimation of the Curie temperature.

Finally, we have shown that the inclusion of long-range interactions does impact the Curie temperature quantitatively; however, the qualitative behavior remains the same. Therefore, the analytical formulas provided in this work can be used to obtain a qualitative estimation of Curie temperatures of $2 \mathrm{D}$ ferromagnets with the least effort. However, for quantitatively more accurate results, one will have to include the long-range interactions and use the exact methods depending on the anisotropy.

\section{ACKNOWLEDGMENTS}

This work was sponsored by the U.S. Department of Defense, Defense Threat Reduction Agency Grant No. HDTRA1-18-1-0018. The content of the information does not necessarily reflect the position or the policy of the federal government, and no official endorsement should be inferred. This work was supported by Imec's Industrial Affiliation Program. This material is based upon work supported by the National Science Foundation under Grant No. 1802166.

S.T., J.V., W.G.V., and B.S. conceived the project. S.T. and J.V. developed the method and the code, and performed the simulations. All the authors analyzed the obtained results. S.T. wrote the paper with all the authors contributing to the discussion and preparation of the manuscript.

\section{APPENDIX}

We briefly discuss the MC, the Green's function, and the RNSW methods in this Appendix.

\section{Renormalized spin-wave method}

First proposed by Bloch et al. [40], spin waves have been used extensively in the theory of magnetism. The framework of the spin waves starts by bosonizing the spin operators $(\hat{\mathbf{S}})$ of the Heisenberg Hamiltonian using the HolsteinPrimakoff transformation [41], with $\hat{S}^{+}=2 S \sqrt{1-\frac{\hat{a}^{\dagger}}{2 S}} \hat{a}^{\dagger}$, $\hat{S}^{-}=2 S \hat{a} \sqrt{1-\frac{\hat{a}^{\dagger} \hat{a}}{2 S}}$, and $\hat{S}^{z}=S-\hat{a}^{\dagger} a$. Here, $\hat{S}^{x}=\frac{\hat{S}^{+}+\hat{S}^{-}}{2}$ and $\hat{S}^{y}=\frac{\hat{S}^{+}-\hat{S}^{-}}{2 i} \cdot \hat{a}^{\dagger} / \hat{a}$ are the bosonic creation/annihilation operators, and $S$ is the spin. We put the value of spin operators and $J$ in Eq. (2b) and ignore the fourth-order terms,

$$
\begin{aligned}
H= & -\sum_{i, j} J_{i j}\left(1-\Delta_{i j}\right) S\left(\hat{a}_{i}^{\dagger} \hat{a}_{j}-\frac{\hat{a}_{i}^{\dagger} \hat{a}_{i}^{\dagger} \hat{a}_{i} \hat{a}_{j}}{2 S}-\frac{\hat{a}_{i}^{\dagger} \hat{a}_{j}^{\dagger} \hat{a}_{j} \hat{a}_{j}}{2 S}\right)+\text { H.c. } \\
& -\frac{1}{2} \sum_{i, j} J_{i j}\left(1+\Delta_{i j}\right)\left(S^{2}-S \hat{a}_{i}^{\dagger} a_{i}+S \hat{a}_{j}^{\dagger} \hat{a}_{j}+\hat{a}_{i}^{\dagger} \hat{a}_{i} \hat{a}_{j}^{\dagger} \hat{a}_{j}\right) .
\end{aligned}
$$

We now make a Hartree-Fock approximation to decouple the second-order terms of Eq. (A1) as $\hat{a}_{i}^{\dagger} \hat{a}_{j}^{\dagger} \hat{a}_{j} \hat{a}_{j}=\hat{a}_{i}^{\dagger} \hat{a}_{j}\left\langle\hat{a}_{j}^{\dagger} \hat{a}_{j}\right\rangle$. The second-order terms are merely the bosonic number density terms $\left(\left\langle\hat{a}_{i}^{\dagger} \hat{a}_{i}\right\rangle=\langle n\rangle\right)$. With these substitutions, we obtain

$$
H=H_{0}\left(1-\frac{\langle n\rangle}{2 S}\right),
$$

with $\quad H_{0}=-\sum_{i, j} J_{i j}(1-\Delta) S\left(\hat{a}_{i}^{\dagger} \hat{a}_{j}\right)+\frac{1}{2} \sum_{i, j} J_{i j}(1+$ $\Delta)\left(S \hat{a}_{i}^{\dagger} \hat{a}_{i}+S \hat{a}_{j}^{\dagger} \hat{a}_{j}\right)+$ H.c. The creation and annihilation operators are transformed in their reciprocal space,

$$
\begin{aligned}
a_{i}^{\dagger} & =\sum_{\mathbf{k} \in B} \exp \left(i \mathbf{k} \cdot r_{i}\right) a^{\dagger}(\mathbf{k}), \\
a_{i} & =\sum_{\mathbf{k} \in B} \exp \left(i \mathbf{k} \cdot r_{i}\right) a(\mathbf{k}) .
\end{aligned}
$$

Substituting Eq. (A3) into Eq. (A2), we obtain the Hamiltonian

$$
H(k)=H_{0}(k)\left(1-\frac{\langle n\rangle}{2 S}\right) .
$$

The eigenvalue of Eq. (A4) is the excitation energy $E_{l}(\mathbf{k}, T)$ for the $l$ th band. Here, $E_{l}(\mathbf{k}, T)$ is temperature dependent because $\langle n(T)\rangle$ follows Bose-Einstein statistics,

$$
\langle n(T)\rangle=\int_{\mathrm{BZ}} \sum_{l} \frac{d^{2} \mathbf{k}}{\exp \left[\frac{E_{l}(\mathbf{k}, T)}{k_{\mathrm{B}} T}\right]-1} .
$$

To calculate the Curie temperature, the starting spin configuration is considered to be pointing in the $z$ direction. The magnetization is defined as $S=\left\langle\hat{S}^{z}\right\rangle$, with $\left\langle\hat{S}^{z}\right\rangle=S-$ $\left\langle a^{\dagger} a\right\rangle$ (Holstein-Primakoff transformation). As defined earlier, $\left\langle a^{\dagger} a\right\rangle=\langle n(T)\rangle$, leading to the equation for magnetization as a function of temperature,

$$
S(T)=S-\frac{1}{N} \int_{\mathrm{BZ}} \sum_{l} \frac{d^{2} \mathbf{k}}{\exp \left[\frac{E_{l}(\mathbf{k}, T)}{k_{\mathrm{B}} T}\right]-1} .
$$

Here, $N$ is the number of atoms in the unit cell, $l$ is the band index, and $S$ is the initial magnetization. Solving magnetization 
$S(T)$ and the energy eigenvalue $E_{l}(\mathbf{k}, T)$ self-consistently, we obtain the temperature-dependent magnetization. However, Eq. (A6) diverges for $E_{l}(\mathbf{k}, T)=0$. To avoid divergence, we define the Curie temperature as the temperature at which $S(T)=S / 2$.

\section{Green's function method}

Zubarev's double-time temperature-dependent Green's functions have been proven successful for three-dimensional ferromagnets in the past, and this over the entire temperature range $[42,43]$. The technique explicitly accounts for the fact that spins obey bosonic commutation relations between distinct lattice sites and fermionic ones between different lattice sites. First, one needs to derive an equation of motion for the Green's functions-this is an exact relation that derives from the Heisenberg equation of motion. The Green's function equation of motion that we derive is

$$
\omega G_{i j}^{\alpha}=\frac{1}{2 \pi}\left\langle\left[\hat{S}_{i}^{\alpha}, \hat{S}_{i}^{-}\right]\right\rangle \delta_{i j}+\left\langle\left\langle\left[\hat{S}_{i}^{\alpha}, \hat{H}\right] ; \hat{S}_{j}^{-}\right\rangle\right\rangle .
$$

Here, $i \omega$ is the excitation energy, $\hat{H}$ is the Heisenberg Hamiltonian, and $G_{i j}^{\alpha}=\left\langle\left\langle\hat{S}_{i}^{\alpha} ; \hat{S}_{j}^{-}\right\rangle\right\rangle$is the Green's function for the spin operator $\hat{S}_{i}^{\alpha}$ with $\alpha \in\{+,-, z\}$. With some algebra, it can be found that the higher-order Green's function $\left\langle\left\langle\left[\hat{S}_{i}^{\alpha}, \hat{H}\right] ; \hat{S}_{j}^{-}\right\rangle\right\rangle$reduces to $\left\langle\left\langle\hat{S}_{i}^{\alpha} \hat{S}_{j}^{\beta} ; \hat{S}_{j}^{-}\right\rangle\right\rangle$, where $\beta \in\{+,-, z\}$. To allow for a solution of the Green's function equation of motion [Eq. (A7)], higher-order Green's functions $\left\langle\left\langle\hat{S}_{i}^{\alpha} \hat{S}_{j}^{\beta} ; \hat{S}_{j}^{-}\right\rangle\right\rangle$are decoupled in terms of lower-order Green's function $G_{i j}^{\alpha}$ using the Tyablikov decoupling approximation [44] (which gives the same results as Englert's random phase approximation combined with the appropriate form of the fluctuation-dissipation theorem [45]). The Tyablikov decoupling scheme decouples higher-order terms using

$$
\begin{aligned}
\left\langle\left\langle\hat{S}_{i}^{\alpha} \hat{S}_{l}^{\beta} ; \hat{S}_{j}^{-}\right\rangle\right\rangle & \rightarrow\left\langle\hat{S}_{l}^{\beta}\right\rangle\left\langle\left\langle\hat{S}_{i}^{\alpha} ; \hat{S}_{j}^{-}\right\rangle\right\rangle+\left\langle\hat{S}_{i}^{\alpha}\right\rangle\left\langle\left\langle\hat{S}_{l}^{\beta} ; \hat{S}_{j}^{-}\right\rangle\right\rangle \\
& =\left\langle\hat{S}_{l}^{\beta}\right\rangle G_{i j}^{\alpha}+\left\langle\hat{S}_{i}^{\alpha}\right\rangle G_{l j}^{\beta} .
\end{aligned}
$$

We then define the homogenous magnetization $M=\left\langle\hat{S}^{z}\right\rangle$ and write the Green's function in the reciprocal space,

$$
G_{i j}^{\alpha}=\frac{1}{N} \sum_{\mathbf{k} \in B} \exp \left[i \mathbf{k} \cdot\left(r_{i}-r_{j}\right)\right] G^{\alpha}(\mathbf{k}) .
$$

Combining Eqs. (A7), (A8), and (A9), we obtain a matrix equation,

$$
[\omega \mathbf{I}-\boldsymbol{\Gamma}(\mathbf{k})] \mathbf{G}(\mathbf{k})=\mathbf{A} .
$$

I is an identity matrix of size $3 \times 3$, and $\mathbf{G}(\mathbf{k})$ is comprised of three Green's functions $\left\{G^{+}, G^{-}, G^{z}\right\}$. For details on building matrices $\Gamma(\mathrm{k})$ and $\mathrm{A}$, the interested reader may refer to Vanherck et al. [29,30].

The Green's function in Eq. (A10) is solved selfconsistently with the homogenous magnetization $M$ for each temperature. The temperature at which the homogenous magnetization $M$ becomes 0 is referred to as the Curie temperature. However, in the absence of an external field, taking the limit of small magnetization (close to the ferromagnetic transition temperature) yields an explicit expression for the Curie temperature. For effective easy-axis anisotropies $\sum_{j} J_{i j} \Delta_{i j} \leqslant$
0 , the Curie temperature vanishes in accordance with the Mermin-Wagner theorem [32]. On the other hand, $T_{\mathrm{C}}$ for effective easy-axis anisotropies can be written as [29,30]

$$
k_{\mathrm{B}} T_{\mathrm{C}}=\frac{S(S+1)}{3 \Phi_{\mathrm{C}}}, \quad \Phi_{\mathrm{C}}=\frac{1}{v_{b}} \int_{\mathrm{BZ}} \phi_{\mathrm{C}}(\mathbf{k}) d \mathbf{k} .
$$

The integrand is

$$
\phi_{\mathrm{C}}(\mathbf{k})=\frac{1}{T-f_{\mathrm{E}}-\left\|f_{\mathrm{O}}\right\|} .
$$

Let $\sum_{n}$ represent a sum over all $n$th neighbors, i.e., NN, NNN, and $\sum_{n, \mathrm{E}}^{n}$ and $\sum_{n, \mathrm{O}}$ the same sum but restricted to atoms located on the same (equal, E) or the other $(\mathrm{O})$ sublattice. We define

$$
\begin{aligned}
T & =\sum_{n} J_{n}\left(1+\Delta_{n}\right), \quad f_{\mathrm{E}}=\sum_{n, \mathrm{E}} F_{n}, \\
\text { and } f_{\mathrm{O}} & =\sum_{n, \mathrm{O}} F_{n} .
\end{aligned}
$$

$T$ is a measure for the total anisotropic exchange interaction. $f_{\mathrm{E}}$ and $f_{\mathrm{O}}$ are defined in terms of $F_{n}=J_{n}$ $\left(1-\Delta_{n}\right) \sum_{p} \exp \left(i \mathbf{k} \cdot \mathbf{r}_{p}\right) \delta_{n p}$. Here, $\delta_{n p}=1$ for $n=p$.

Compared to the renormalized spin-wave theory, not only the excitation energies but also the effective density of states are renormalized by the magnetization, yielding a better description over the entire temperature range. The major difference between the Green's function and the RNSW methods lies in the level at which the decoupling is performed. The Tyablikov decoupling [44] is performed at the level of spin operators, whereas the Hartree-Fock decoupling is performed at the level of bosonized spin waves.

\section{Monte Carlo method}

For both the Ising and the Monte Carlo methods with anisotropy, Eq. (2b) is treated as a classical equation with spin operators $(\hat{\mathbf{S}})$ becoming spin vectors $(\mathbf{S})$. We use the Metropolis algorithm to simulate the phase change of the classical Heisenberg Hamiltonian [46].

For the Metropolis sampling of the Ising and Monte Carlo methods, the spin vectors become scalars and are fixed to take values $S \in\left\{-S_{\max }, S_{\max }\right\}$. Whereas for the Monte Carlo method with anisotropy, the spin vectors are sampled using a spherical sampling scheme [24]. From the Metropolis algorithm, we obtain the magnetic susceptibility and specific heat as a function of temperature. We obtain the Curie temperature from the peak of specific heat or susceptibility as they both coincide for easy-axis ferromagnets.

\section{DFT calculations}

The $J, \Delta$, and $S$ were directly obtained from the C2DB database and were fed into the analytical formulas. However, we calculated the long-range $J$ parameters for $\mathrm{Cr}$ compounds and $\mathrm{Fe}_{2} \mathrm{~F}_{2}$, using the method developed in Ref. [24], which uses noncollinear DFT calculations. All the $a b$ initio DFT calculations reported in this work were performed using the Vienna $a b$ initio simulation package (VASP) [47,48]. The ground-state self-consistent field (SCF) calculations were 
performed using a projector-augmented wave (PAW) potential [47] with a generalized-gradient approximation as proposed by Perdew-Burke-Ernzerhof (PBE) [49]. We have used a kinetic energy cutoff of $400 \mathrm{eV}$ for our DFT calculations. The Brillouin zones were sampled using a $\Gamma$-centered $k$-point mesh of size $5 \times 5 \times 1$ points for $2 \times 1 \times 1$ supercells.
The Cr-compound supercells were relaxed until the force on each of the ions was below $10 \mathrm{meV} / \AA$. The energy convergence criterion for the subsequent SCF calculations was set to $10^{-4} \mathrm{eV}$. The C2DB parameters and our own DFT calculations for the $\mathrm{Cr}$ compounds and $\mathrm{Fe}_{2} \mathrm{~F}_{2}$ showed a difference of less than $20 \%$ in the $J$ parameters.
[1] B. Huang, G. Clark, E. Navarro-Moratalla, D. R. Klein, R. Cheng, K. L. Seyler, D. Zhong, E. Schmidgall, M. A. McGuire, D. H. Cobden, W. Yao, D. Xiao, P. Jarillo-Herrero, and X. Xu, Layer-dependent ferromagnetism in a van der Waals crystal down to the monolayer limit, Nature (London) 546, 270 (2017).

[2] Z. Zhang, J. Shang, C. Jiang, A. Rasmita, W. Gao, and T. $\mathrm{Yu}$, Direct photoluminescence probing of ferromagnetism in monolayer two-dimensional $\mathrm{CrBr}_{3}$, Nano Lett. 19, 3138 (2019).

[3] C. Gong, L. Li, Z. Li, H. Ji, A. Stern, Y. Xia, T. Cao, W. Bao, C. Wang, Y. Wang, Z. Q. Qiu, R. J. Cava, S. G. Louie, J. Xia, and $\mathrm{X}$. Zhang, Discovery of intrinsic ferromagnetism in twodimensional van der Waals crystals, Nature (London) 546, 265 (2017).

[4] D. L. Cortie, G. L. Causer, K. C. Rule, H. Fritzsche, W. Kreuzpaintner, and F. Klose, Two-dimensional magnets: Forgotten history and recent progress towards spintronic applications, Adv. Funct. Mater. 30, 1901414 (2020).

[5] Z. Wang, I. Gutiérrez-Lezama, N. Ubrig, M. Kroner, M. Gibertini, T. Taniguchi, K. Watanabe, A. Imamoğlu, E. Giannini, and A. F. Morpurgo, Very large tunneling magnetoresistance in layered magnetic semiconductor $\mathrm{CrI}_{3}$, Nat. Commun. 9, 2516 (2018).

[6] D. Zhong, K. L. Seyler, X. Linpeng, R. Cheng, N. Sivadas, B. Huang, E. Schmidgall, T. Taniguchi, K. Watanabe, M. A. McGuire, W. Yao, D. Xiao, K.-M. C. Fu, and X. Xu, Van der Waals engineering of ferromagnetic semiconductor heterostructures for spin and valleytronics, Sci. Adv. 3, e1603113 (2017).

[7] D. Amoroso, P. Barone, and S. Picozzi, Spontaneous skyrmionic lattice from anisotropic symmetric exchange in a Ni-halide monolayer, Nat. Commun. 11, 5784 (2020).

[8] Q. Tong, F. Liu, J. Xiao, and W. Yao, Skyrmions in the Moiré of van der Waals 2D magnets, Nano Lett. 18, 7194 (2018).

[9] Z. Fei, B. Huang, P. Malinowski, W. Wang, T. Song, J. Sanchez, W. Yao, D. Xiao, X. Zhu, A. F. May, W. Wu, D. H. Cobden, J.-H. Chu, and X. Xu, Two-dimensional itinerant ferromagnetism in atomically thin $\mathrm{Fe}_{3} \mathrm{GeTe}_{2}$, Nat. Mater. 17, 778 (2018).

[10] M. Bonilla, S. Kolekar, Y. Ma, H. C. Diaz, V. Kalappattil, R. Das, T. Eggers, H. R. Gutierrez, M.-H. Phan, and M. Batzill, Strong room-temperature ferromagnetism in $\mathrm{VSe}_{2}$ monolayers on van der Waals substrates, Nat. Nanotechnol. 13, 289 (2018).

[11] P. M. Coelho, K. Nguyen Cong, M. Bonilla, S. Kolekar, M.-H. Phan, J. Avila, M. C. Asensio, I. I. Oleynik, and M. Batzill, Charge density wave state suppresses ferromagnetic ordering in $\mathrm{VSe}_{2}$ monolayers, J. Phys. Chem. C 123, 14089 (2019).

[12] J. Feng, D. Biswas, A. Rajan, M. D. Watson, F. Mazzola, O. J. Clark, K. Underwood, I. Marković, M. McLaren, A. Hunter, D. M. Burn, L. B. Duffy, S. Barua, G. Balakrishnan, F. Bertran, P. Le Fèvre, T. K. Kim, G. van der Laan, T. Hesjedal, P. Wahl et al., Electronic structure and enhanced charge-density wave order of monolayer VSe $\mathrm{V}_{2}$, Nano Lett. 18, 4493 (2018).

[13] Y. Zhu, X. Kong, T. D. Rhone, and H. Guo, Systematic search for two-dimensional ferromagnetic materials, Phys. Rev. Mater. 2, 081001(R) (2018).

[14] P. D. Reyntjens, S. Tiwari, M. L. V. de Put, B. Sorée, and W. G. Vandenberghe, Magnetic properties and critical behavior of magnetically intercalated $\mathrm{WSe}_{2}$ : a theoretical study, 2D Mater. 8, 025009 (2020).

[15] R. Mishra, W. Zhou, S. J. Pennycook, S. T. Pantelides, and J.-C. Idrobo, Long-range ferromagnetic ordering in manganesedoped two-dimensional dichalcogenides, Phys. Rev. B 88, 144409 (2013).

[16] A. Ramasubramaniam and D. Naveh, Mn-doped monolayer $\mathrm{MoS}_{2}$ : An atomically thin dilute magnetic semiconductor, Phys. Rev. B 87, 195201 (2013).

[17] S. Tiwari, M. L. Van de Put, B. Sorée, and W. G. Vandenberghe, Magnetic order and critical temperature of substitutionally doped transition metal dichalcogenide monolayers, npj 2D Mater. Appl. 5, 54 (2021).

[18] W. Liu, M. R. Osanloo, X. Wang, S. Li, N. Dhale, H. Wu, M. L. Van de Put, S. Tiwari, W. G. Vandenberghe, and B. Lv, New Verbeekite-type polymorphic phase and rich phase diagram in the $\mathrm{PdSe}_{2-x} \mathrm{Te}_{x}$ system, Phys. Rev. B 104, 024507 (2021).

[19] P. D. Reyntjens, S. Tiwari, M. L. Van de Put, B. Sorée, and W. G. Vandenberghe, Ab-initio study of magnetically intercalated platinum diselenide: The impact of platinum vacancies, Materials 14, 4167 (2021).

[20] D. Torelli, H. Moustafa, K. W. Jacobsen, and T. Olsen, High-throughput computational screening for two-dimensional magnetic materials based on experimental databases of threedimensional compounds, npj Comput. Mater. 6, 158 (2020).

[21] D. Torelli, K. S. Thygesen, and T. Olsen, High throughput computational screening for 2D ferromagnetic materials: the critical role of anisotropy and local correlations, 2D Mater. 6, 045018 (2019).

[22] A. Kabiraj, M. Kumar, and S. Mahapatra, High-throughput discovery of high Curie point two-dimensional ferromagnetic materials, npj Comput. Mater. 6, 35 (2020).

[23] S. Haastrup, M. Strange, M. Pandey, T. Deilmann, P. S. Schmidt, N. F. Hinsche, M. N. Gjerding, D. Torelli, P. M. Larsen, A. C. Riis-Jensen, J. Gath, K. W. Jacobsen, J. J. Mortensen, T. Olsen, and K. S. Thygesen, The computational 2D materials database: high-throughput modeling and discovery of atomically thin crystals, 2D Mater. 5, 042002 (2018).

[24] S. Tiwari, M. L. Van de Put, B. Sorée, and W. G. Vandenberghe, Critical behavior of the ferromagnets $\mathrm{CrI}_{3}, \mathrm{CrBr}_{3}$, and $\mathrm{CrGeTe}_{3}$ and the antiferromagnet $\mathrm{FeCl}_{2}$ : A detailed first-principles study, Phys. Rev. B 103, 014432 (2021). 
[25] X. Lu, R. Fei, and L. Yang, Curie temperature of emerging two-dimensional magnetic structures, Phys. Rev. B 100, 205409 (2019).

[26] L. Webster and J.-A. Yan, Strain-tunable magnetic anisotropy in monolayer $\mathrm{CrCl}_{3}, \mathrm{CrBr}_{3}$, and $\mathrm{CrI}_{3}$, Phys. Rev. B 98, 144411 (2018).

[27] D. Torelli and T. Olsen, Calculating critical temperatures for ferromagnetic order in two-dimensional materials, 2D Mater. 6, 015028 (2018).

[28] T. Olsen, Theory and simulations of critical temperatures in $\mathrm{CrI}_{3}$ and other 2D materials: easy-axis magnetic order and easyplane Kosterlitz-Thouless transitions, MRS Commun. 9, 1142 (2019).

[29] J. Vanherck, B. Sorée, and W. Magnus, Anisotropic bulk and planar Heisenberg ferromagnets in uniform, arbitrarily oriented magnetic fields, J. Phys.: Condens. Matter 30, 275801 (2018).

[30] J. Vanherck, C. Bacaksiz, B. Sorée, M. V. Milošević, and W. Magnus, 2D ferromagnetism at finite temperatures under quantum scrutiny, Appl. Phys. Lett. 117, 052401 (2020).

[31] J. L. Lado and J. Fernández-Rossier, On the origin of magnetic anisotropy in two dimensional $\mathrm{CrI}_{3}, 2 \mathrm{D}$ Mater. 4, 035002 (2017).

[32] N. D. Mermin and H. Wagner, Absence of Ferromagnetism or Antiferromagnetism in One- or Two-Dimensional Isotropic Heisenberg Models, Phys. Rev. Lett. 17, 1133 (1966).

[33] J. A. Sears, M. Songvilay, K. W. Plumb, J. P. Clancy, Y. Qiu, Y. Zhao, D. Parshall, and Y.-J. Kim, Magnetic order in $\alpha-\mathrm{RuCl}_{3}$ : A honeycomb-lattice quantum magnet with strong spin-orbit coupling, Phys. Rev. B 91, 144420 (2015).

[34] K. W. Plumb, J. P. Clancy, L. J. Sandilands, V. V. Shankar, Y. F. $\mathrm{Hu}, \mathrm{K} . \mathrm{S}$. Burch, H.-Y. Kee, and Y.-J. Kim, $\alpha-\mathrm{RuCl}_{3}$ : A spinorbit assisted Mott insulator on a honeycomb lattice, Phys. Rev. B 90, 041112(R) (2014).

[35] H.-S. Kim, Vijay Shankar V., A. Catuneanu, and H.-Y. Kee, Kitaev magnetism in honeycomb $\mathrm{RuCl}_{3}$ with intermediate spinorbit coupling, Phys. Rev. B 91, 241110(R) (2015).

[36] M. Bander and D. L. Mills, Ferromagnetism of ultrathin films, Phys. Rev. B 38, 12015 (1988).
[37] See Supplemental Material at http://link.aps.org/supplemental/ 10.1103/PhysRevResearch.3.043024 for the information regarding the Curie temperature as a function of exchange anisotropy for square and hexagonal lattices, and the full-table for 34 screened 2D magnets containing their Curie temperature calculated using the three methods.

[38] J. Cenker, B. Huang, N. Suri, P. Thijssen, A. Miller, T. Song, T. Taniguchi, K. Watanabe, M. A. McGuire, D. Xiao, and X. Xu, Direct observation of two-dimensional magnons in atomically thin $\mathrm{CrI}_{3}$, Nat. Phys. 17, 20 (2021).

[39] M. A. McGuire, G. Clark, S. KC, W. M. Chance, G. E. Jellison, V. R. Cooper, X. Xu, and B. C. Sales, Magnetic behavior and spin-lattice coupling in cleavable van der Waals layered $\mathrm{CrCl}_{3}$ crystals, Phys. Rev. Mater. 1, 014001 (2017).

[40] F. Bloch, On the theory of ferromagnetism, Z. Phys. 61, 206 (1930).

[41] T. Holstein and H. Primakoff, Field dependence of the intrinsic domain magnetization of a ferromagnet, Phys. Rev. 58, 1098 (1940).

[42] D. N. Zubarev, Double-time green functions in statistical physics, Sov. Phys. Usp. 3, 320 (1960).

[43] H. B. Callen, Green function theory of ferromagnetism, Phys. Rev. 130, 890 (1963).

[44] S. Tyablikov, Retarded and advanced Green functions in the theory of ferromagnetism, Ukrain. Math. J. 11, 287 (1959).

[45] F. Englert, Theory of a Heisenberg Ferromagnet in the Random Phase Approximation, Phys. Rev. Lett. 5, 102 (1960).

[46] N. Metropolis and S. Ulam, The Monte Carlo method, J. Am. Stat. Assoc. 44, 335 (1949).

[47] P. E. Blöchl, Projector augmented-wave method, Phys. Rev. B 50, 17953 (1994).

[48] G. Kresse and J. Furthmüller, Efficient iterative schemes for $a b$ initio total-energy calculations using a plane-wave basis set, Phys. Rev. B 54, 11169 (1996).

[49] J. P. Perdew, K. Burke, and M. Ernzerhof, Generalized Gradient Approximation Made Simple, Phys. Rev. Lett. 77, 3865 (1996). 